\section{Oxidising mouthwash minimises airborne infection risk}

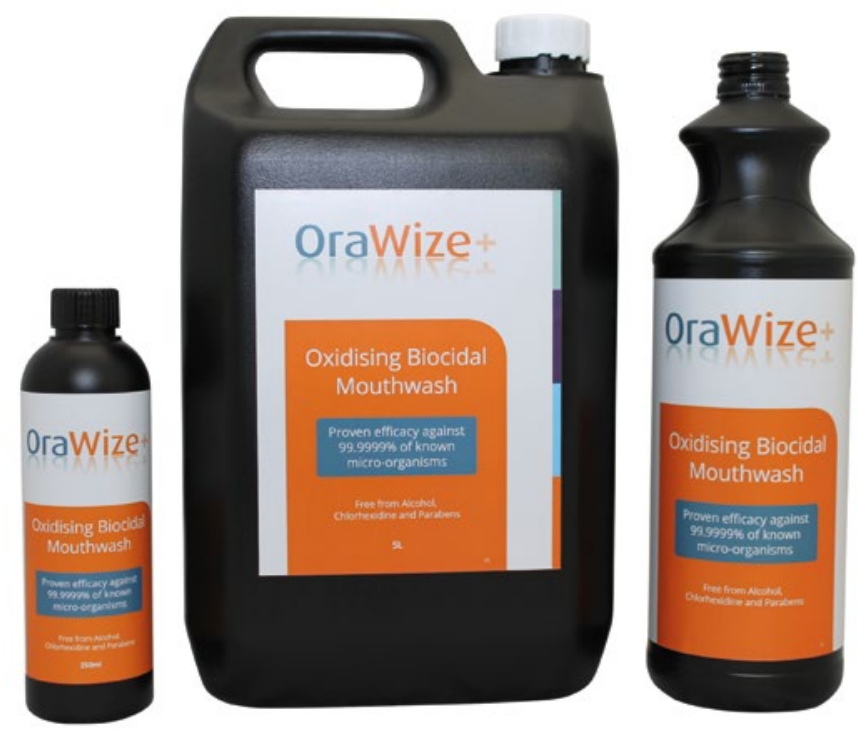

CleanCert Ltd, a Wiltshire-based SME, which develops innovative dental products, is highlighting the oxidising properties of its mouthwash, OraWize+, to assist front-line dental teams in minimising airborne infection risk during the current COVID-19 pandemic.

The unique mouthwash has been developed to both reduce the risk posed by aerosol generating procedures as well as to assist biofilm management during general and specialist dental treatment.

It can be used as a preprocedural rinse, as well as an irrigant during endodontic, periodontal and peri-implantitis treatment, and for post-treatment care by patients to reduce oral microbial load and hence reduce swelling.

While infection control is paramount for dental practices at all times, the current pandemic and national state of emergency has meant that awareness around effective measures of infection control and minimising airborne risk are at an all-time high and that dental teams have a vital role to play in preventing transmission.

Rinsing with an effective biocidal mouthwash immediately prior to any treatment has been shown to reduce the number of microbes found in the aerosol created by dental procedures with a recent study suggesting that an oxidising mouthwash would be more effective.

OraWize+ is a highly oxidising solution that physically destroys harmful micro-organisms by disrupting their RNA (like DNA) which in turn kills their cell structure. It is $99.9999 \%$ effective against known pathogens and is also free from alcohol, chlorhexidine and parabens. It is also non-toxic and has a very low order irritant in eyes, skin and even with ingestion, so the risk is minimised even if the patient accidentally swallows it.

OraWize+ oxidising mouthwash has a highly effective oxidising action: this property has been shown in studies from Wuhan to be required for the effective killing of coronavirus in the mouth and in aerosols.

The product's active ingredient has also been tested against the stringent BS EN 14476 , which is a quantitative suspension test to evaluate virucidal activity in nonenveloped viruses, such as norovirus, and more friable enveloped viruses, such as HIV, influenza and coronaviruses. It achieved this with a one-minute contact time.

Because of these properties, CleanCert has decided to support the national COVID-19 response by temporarily making the mouthwash irrigant available in 5 litre quantities for use as an oxidising biocidal mouthwash by dental teams offering emergency dental care. And by cutting the 5 $\mathrm{L}$ bottle price in half, it aims to help ease the financial burden for the NHS and those on the dental frontline.

OraWize+ is available from The Dental Directory, Denka UK and 360 Dental.

CleanCert is offering $50 \%$ off for $5 \mathrm{~L}$ containers to all NHS 'Urgent Dental Care Hubs', via the distributors above. Terms and conditions apply.

For further info, contact technical@ cleancert.co.uk or call 08443511115.

\section{Cola lime flavoured desensitising varnish}

As essential in every practice as forceps and drills: VOCO Profluorid Varnish (VPV). The popular fluoridecontaining varnish for desensitising teeth is now also available in cola lime flavour - giving patients even more choice. The tang of cola combined with the fruitiness of lime appeals to a wide range of patients and is the perfect complement to the existing portfolio. In total, there are now six flavours available: melon, mint, cherry, caramel, bubble gum and cola lime.

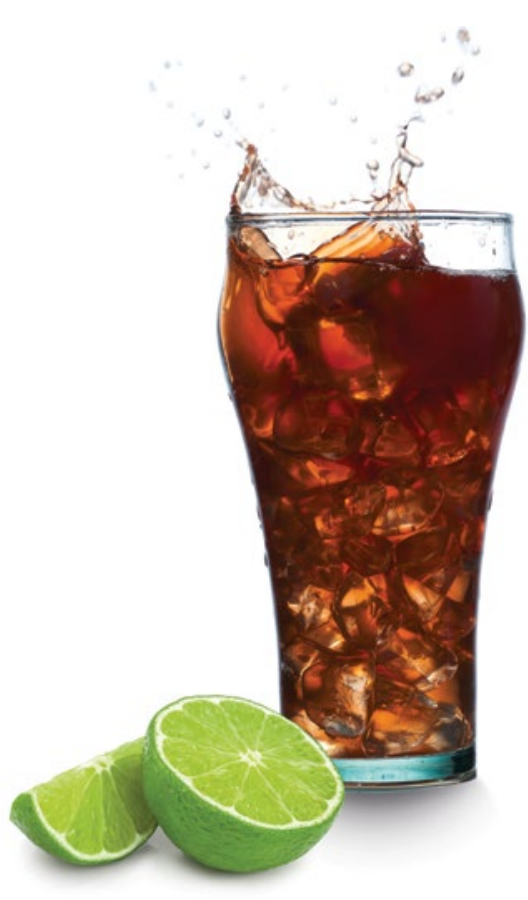

VOCO Profluorid Varnish is ideal for the treatment of hypersensitive teeth and for sealing the dentinal tubules during cavity preparation or in cases of sensitive root surfaces. Treatment with VPV following cleaning and polishing is also a sensible precaution in order to refill any eroded calcium fluoride depots. The fluoride content is 22,600 ppm fluoride (approx. 5\% sodium fluoride). In addition, the whitetransparent varnish impresses with high moisture tolerance and excellent adhesion to dental hard tissue. Like all the other members of the VPV family, VOCO Profluorid Varnish cola lime is available in the practical SingleDose and the $10 \mathrm{ml}$ tube. 